\title{
Necrotizing pancreatic pseudocyst induced by percutaneous transhepatic cholangiography \& endoscopic retrograde cholangiography for biliary strictures after liver transplantation: Case report
}

\author{
Mi Rin LEE, Jae Do YANG, Sung Woo AHN, Hee Chul YU*
}

Department of General Surgery, Jeonbuk National University Hospital, Jeonju, Korea

Introduction: Biliary strictures that developed after LT are managed with endoscopic retrograde cholangiography (ERC), percutaneous transhepatic cholangiography (PTC), or surgical revision. In cases where conventional endoscopic access is failed, alternative treatments should be achieved by means of the rendezvous technique, which is a hybrid technique combining PTC and ERC. We describe a rare case of necrotic pseudocyst occurring after the Rendezvous technique for treatment of post-transplant biliary strictures.

Methods: A case review has been done.

Results: A 65-year-old man who presented with hepatocellular carcinoma complicating chronic hepatitis B liver cirrhosis underwent live donor liver transplantation (LDLT) from his son. One year after, he presented mild pruritus. The serum liver enzymes and bilirubin level raised but, liver biopsy was not diagnostic of rejection. ERCP showed stricture of the biliary anastomosis and tried to PTC and ERC, but failed three times. Cannulation of a biliary stricture can be achieved by the Rendezvous technique with progressive balloon dilation and the placement of two plastic stents. The patient was readmitted 2 weeks later with pain and vomiting with fever. CT showed $17 \mathrm{~cm}$ large pancreatic necrotic pseudocyst which was possibly a complication of previous intervention. Percutaneous drainage (PCD) of the collection yielded abscess. Despite of drainage and antibiotics management during 4 weeks, symptoms and pancreatitis were not resolved. After repositioning of PCD and irrigating with saline daily, symptoms including fever have improved.

Conclusions: Necrotizing pancreatic pseudocyst has been happened rarely as a complication after post-ERCP in liver transplantation patients. and needed intensive treatments and long-term hospitalization for preventing fatal course. 